\title{
Ion channels in mechanosensing and electrical signaling in plants
}

\author{
Jean-Marie Frachisse ${ }^{1, a}$ and Daniel $\operatorname{Tran}^{1}$ \\ ${ }^{1}$ Institute for Integrative Biology of the Cell (I2BC), CEA, CNRS, Univ Paris-Sud, Université Paris-Saclay, \\ SciencesPlant Saclay, 91198, Gif-sur-Yvette cedex, France
}

\begin{abstract}
Plants as animals have cell membrane equipped with ion channels. These channels embedded in the membrane allowed ion to move in or out the cell. Therefore these proteins are involved in several physiological processes such as nutrition, sensing (of environmental factors) and long-distance communication. In this "slide presentation" we report the possible role of ion channels in sensing oscillation and in long distance signalling in plant.

The first example concerns the mechanosensitive channels of the MSL (Mechanosensitive channel Small conductance-Like) family and illustrate how it possibly behave as an oscillation sensor for the plant. The function of such a channel is consistent with the capacity of terrestrial plants to react to repetitive mechanical load produced by wind.

The second example concerns the role of mechano-gated and voltage-gated channels in the generation and long distance propagation of electrical signal in plant. More precisely, it illustrate how Action Potential and Slow Wave of depolarization are generated and propagate along plant tissues. Eventually the relevance of such electrical signals in plant is illustrated by two examples.
\end{abstract}

\footnotetext{
${ }^{\mathrm{a}}$ Corresponding author: jean-marie.frachisse@,i2bc.paris-saclay.fr
} 